\title{
FMH und SBK unterstützen Motion über die Patientensicherheit und die Garantie der Behandlungsqualität
}

\author{
Brief an die Nationalrätinnen und -räte
}

Sehr geehrte Damen und Herren

Die Verbindung der Schweizer Ärztinnen und Ärzte (FMH) und der Schweizer Berufsverband der Pflegefachfrauen und Pflegefachmänner (SBK) drücken hiermit ihre Unterstützung für die obgenannte Motion aus. Diese wird zunächst innerhalb der parlamentarischen Fraktionen diskutiert, bevor sie dem Plenum zur Beratung vorgelegt wird.

Als Ärztinnen und Ärzte und Pflegefachfrauen und -männer möchten wir abermals unsere Bereitschaft betonen, uns aktiv an der Erarbeitung und Realisierung von Projekten und Massnahmen zu beteiligen, die das Fehlerrisiko in der Behandlung mindern und deren Qualität verbessern.

Die in der Motion vorgeschlagene Plattform stellt aus unserer Sicht eine äusserst konstruktive Lösung dar, um auf dem richtigen Weg voranzukommen. Die Sicherheit der Patientinnen und Patienten ist ein berechtigtes Anliegen, welches gemeinsam von allen Akteuren im Gesundheitswesen getragen werden muss. Dazu gehören sowohl die Leistungserbringer als auch die politischen Entscheidungsträger!

Pierre Théraulaz, Präsident SBK

Urs Weyermann, Geschäftsführer SBK

NB. Der Nationalrat hat am 3. März 2005 die Motion seiner Kommission für soziale Sicherheit und Gesundheit behandelt und sie mit $85 \mathrm{zu}$ 35 Stimmen gegen den Willen der Regierung überwiesen. Bundesrat Pascal Couchepin sagte dabei unter anderem, er anerkenne die Notwen-
Deshalb sind wir um so überraschter, dass der Bundesrat die Schaffung dieser Plattform als «nicht prioritär» betrachtet.

Die in der Antwort des Bundesrates vom 23. Februar vorgeschlagenen «Polizeimassnahmen» («Ergreifung und Stärkung der notwendigen Massnahmen auf der Grundlage des Art. 58 KVG») sind unseres Erachtens weit davon entfernt, die Bedürfnisse in diesem Bereich von brennender Aktualität zu befriedigen: Die Qualität auf diese Weise «verordnen» zu wollen ist eine offensichtliche Illusion, von welcher sich nur die Bundesverwaltung täuschen lässt.

Hingegen scheint uns die Verankerung der Patientensicherheit im KVG als Verantwortung des Bundes und die Perspektive, die erforderlichen Massnahmen gemeinsam zu planen und anschliessend umzusetzen, ein vielversprechender Weg zu sein.

Sicherheit und Qualität der Behandlung sind ein Bereich, wo einseitige Massnahmen zum Scheitern verurteilt sind und vorhandene Absprachemöglichkeiten allseits unterstützt werden müssen. Die FMH und der SBK wären Ihnen deshalb äusserst dankbar, wenn Sie diese Motion der SGK-NR unterstützen würden.

Jacques de Haller, Präsident FMH

Yves Guisan, Nationalrat, Vizepräsident FMH

digkeit, die Qualität der medizinischen Behandlung sicherzustellen. Die alleinige Verantwortung des Bundes für Qualitätssicherung und Patientensicherheit sei jedoch weder sinnvoll noch finanzierbar. 